\title{
The realization of temperature range control strategy on household air heater
}

\author{
Wu Jian \\ Business school \\ Beijing Institute of Fashion Technology \\ Beijing, China \\ wujian189@outlook.com
}

\author{
Liu Liang \\ Business school \\ Beijing Institute of Fashion Technology \\ Beijing, China \\ 1711106980@qq.com
}

Abstract-This design adopts STC89C52RC MCU, utilizes DS18B20 digital temperature sensor, VS1838B infrared receiver, and combines the temperature range control method to realize intelligent heating fan. The test results show that the system can run independently and continuously after setting the temperature value, and keep the room temperature near the upper limit of the temperature, achieving the effect similar to air conditioning. This function innovation is very meaningful.

Keywords-intelligent air heater; STC89C52; DS18B20; VS1838B

\section{OVERALL SYSTEM WORKFLOW}

During the operation of the system, a temperature range should be set first, which serves as the basis for controlling the heater. The single chip computer receives the real-time data of the temperature sensor and compares it with the set value to make a judgment. The judgment results are used to control the output level of the heater, which are: level 1, level 2 and stop. When timer time comes, the whole heater stops running. The infrared remote controller can set working mode and set temperature upper and lower limit and timing time. The workflow is shown in Fig. 1 below:

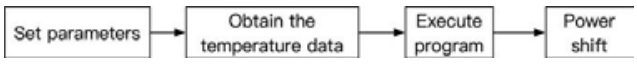

Fig. 1. System workflow

\section{HARDWARE DESIGN}

The system is mainly composed of MCU, keys, temperature sensor, infrared receiver, heating module controlled by electromagnetic valve and LCD display. Temperature upper and lower limit can be set by buttons and infrared remote control. The temperature sensor is mainly responsible for collecting the temperature and returning the value to the MCU. The MCU compares the current temperature with the set upper and lower temperature, and gives the shift size that should be output. The setting of related parameters is displayed by LCD. The overall hardware block diagram is shown in Fig.2:

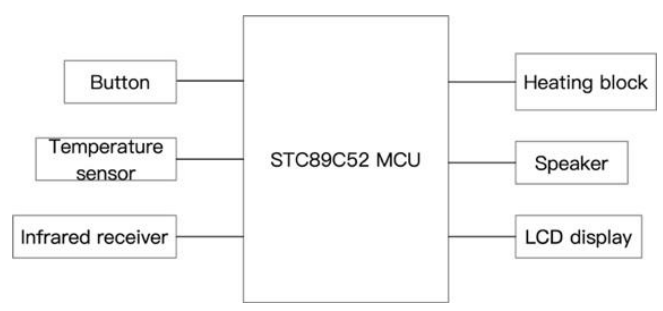

Fig. 2. Hardware block diagram

The circuit schematic diagram of the whole heater system is shown in Fig. 3 below. In addition to the MCU's minimum system, infrared reception circuit, key circuit, temperature detection circuit, alarm circuit, liquid crystal display circuit and relay control circuit are used here. Since this paper mainly introduces the application of temperature range 
control, the following part mainly introduces several core circuits, while the other part does not give more explanations.

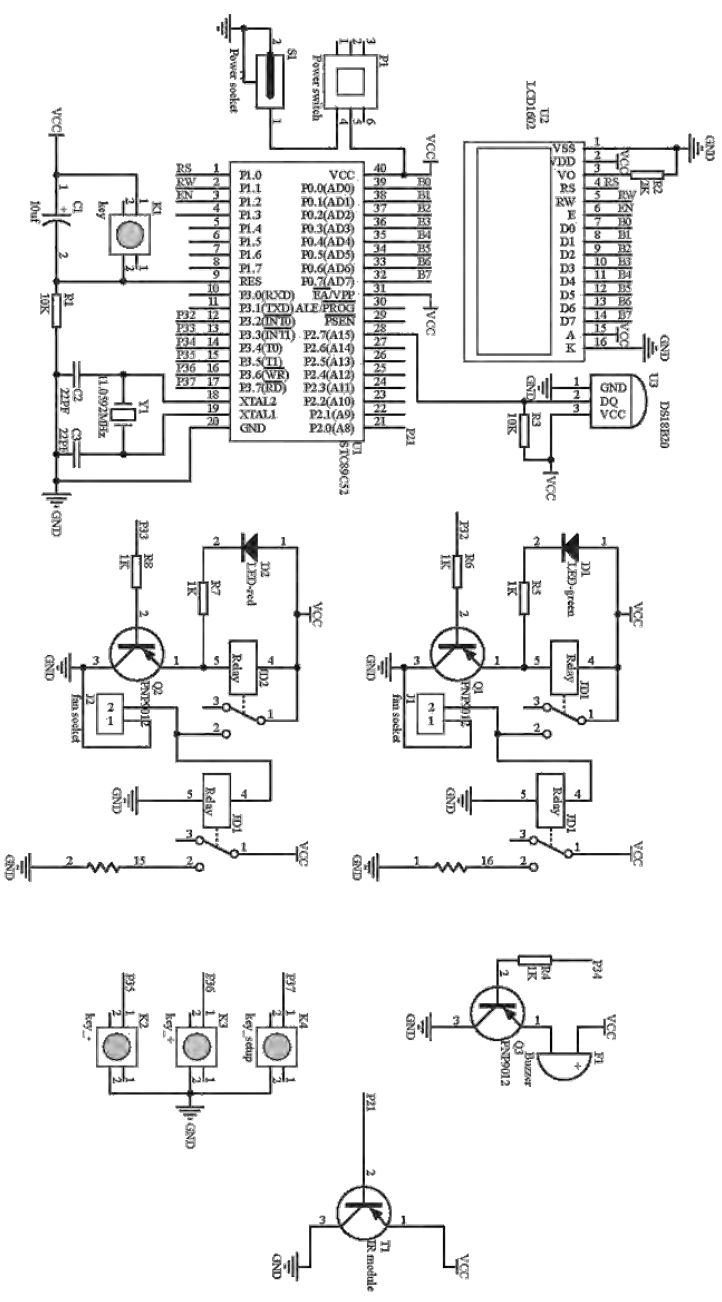

Fig. 3. Electrical schematic of heater

\section{A. Relay control circuit}

In this system, since the dc fan is used, its current is very large compared to the integrated circuit of single chip microcomputer, so relay is adopted to reduce the voltage fluctuation caused by fans on and off.

There are relays to control the heating wire and the fan. The current flows through the coil of the relay, which makes the armature absorb and conduct the circuit under the action of electromagnetic effect. However, since the output capacity of the single chip is relatively weak, 9012PNP triode is needed to drive it. Single chip microcomputer can not directly control the relay, but can control the pipe. The transistor here acts as a "bridge". The resistance in series with LED is the partial pressure current limiting resistance. The base of the pipe is connected with the single chip computer, which only needs to control the high and low level of IO port of the single chip computer to control the pipe on and off. A diode should also be added to a standard relay circuit to release the electrical energy in the relay to prevent the pipe from breaking through. But since the reverse electromotive force of the relay is not very large, it is not added here for simplicity. The relay switch that controls the heating wire is the same logic as the fans. Here, an external $220 \mathrm{~V}$ power supply is used to drive the heating wire. The relay controlling the wire and the relay controlling the fan operate synchronously, so it is not shown in the circuit diagram.

\section{B. Temperature detecting circuit}

The temperature is detected using a DS18B20 sensor. Line 2 is connected to a single chip for the transmission of temperature data, line 1 is grounded, and line 3 is connected to a $5 \mathrm{~V}$ power supply. Resistance is usually added between lines 2 and 3 to increase output ability.

\section{LCD display circuit}

Since only temperature, output level and timing time are the display characters involved in the heater system, LCD1602 can be fully met. Connect 5V power supply, and then connect 8 data cables to $\mathrm{P} 0$ port of the microcontroller. The effect of the display is adjusted by the potential connected to VO port. After comprehensive analysis, the resistance value of $2 \mathrm{~K}$ can be used to achieve better display effect. The three lines of 4,5 and 6 are the functional control end of the display screen, and 15 and 16 control backlight.

\section{THE PROGRAM DESIGN}

\section{A. Main program design}

The main program is inspired by the human body. The main program is the head, and the subroutines are our limbs and organs. The head can control the movement of each part, and each part can communicate with the head and exchange 
information. The software topology of the whole system is shown in Fig.4:

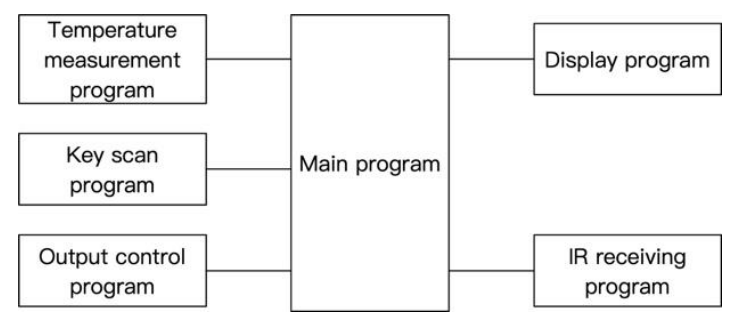

Fig. 4. The software block diagram

Firstly, program initialization is carried out after the system is energized, in which various initialization subroutines are performed, respectively initialization of infrared ray reception, LCD initialization, temperature reading initialization, timer initialization and buzzer initialization. All initializers are outside of while (1), which is initiated at startup and executed only once. While (1) is an infinite loop program, which is what the MCU is doing all the time. As the operation process of the heater needs to respond to the button and the command of the remote control at any time, the key detection should be in it. The next step is to determine the current running mode. Mode 0 is normal operation mode. Only in this mode can temperature data be read and control of output gear is implemented. Mode 1 is to set the temperature upper and lower limit, and mode 2 is to set the timing time. In both modes, the heater stops working. Pressing the Settings button will switch the mode in turn. Mode 1 and 2 are placed in subfunctions and called only in the main function. When the temperature value detected by the single chip microcomputer is less than the temperature value minimum limit, start level 2 heating; When the detected temperature value is between the upper and lower limit of the set temperature, start level 1 heating; Stop heating when the detected temperature is greater than the set temperature. Both the mode and timer have flags that perform related operations when the corresponding flag is a defined value. The flow chart of the main program of the system is shown in Fig.5:

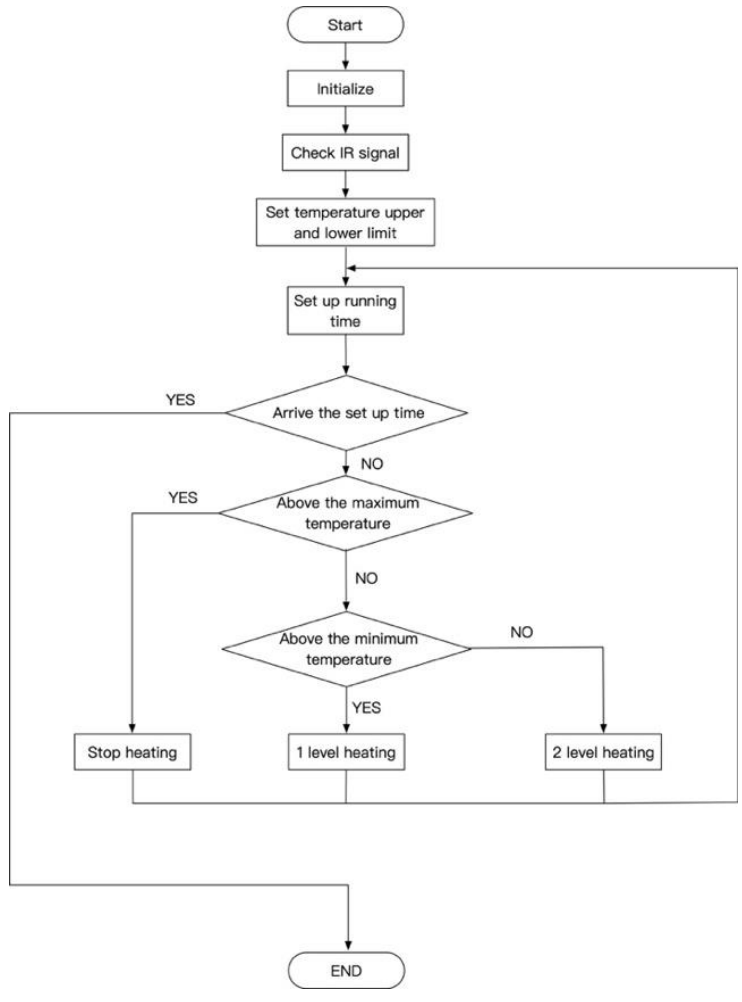

Fig. 5. Main program flow chart

\section{B. Temperature interval control logic}

For the heating fan system, the controlled object -- the temperature is not as high as the requirements of boilers, fermentation containers and so on, which only needs simple adjustment. Therefore, output level adjustment is adopted instead of PID control.

First, there are two parameters that need to be set by the user, namely the upper and lower limit of temperature. For convenience, let's say that the lower limit is A and the upper limit is B. When the room temperature is less than $\mathrm{A}$, the heating fan system opens the high-power(level 2) heating mode. When the temperature is between $\mathrm{A}$ and $\mathrm{B}$, turn on the low-power(level 1) heating mode; When the temperature is higher than B, stop heating. As shown in table I below:

TABLE I. OUTPUT LEVEL CONTROL LOGIC

\begin{tabular}{|c|c|c|c|}
\hline Temperature & $\begin{array}{c}\text { Less than } \\
\text { A }\end{array}$ & $\begin{array}{c}\text { Between A } \\
\text { and B }\end{array}$ & $\begin{array}{c}\text { Greater } \\
\text { than B }\end{array}$ \\
\hline Heating level & 2 & 1 & 0 \\
\hline
\end{tabular}




\section{Shift control programming}

In the output control system of the warm air blower, two relays are used to control two heating fans respectively. The first is the acquisition of the current temperature. When the temperature value is greater than the set upper limit value, the power supply of the fan will be disconnected to stop the heating fan. When the temperature value is between the upper and lower limit of the set temperature, control the suction of one of the relays to operate a heating fan. When the temperature value is less than the set lower limit value, control two fans to energize, so that two heating fans can operate at the same time. The temperature control subroutine flow chart is shown in Fig.6.

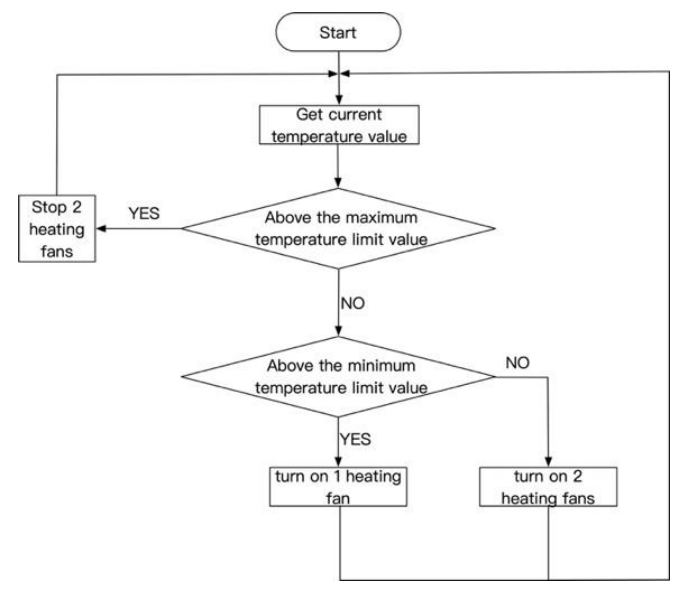

Fig. 6. Temperature control subroutine flow chart

The temperature value comes from the ROM of DS18B20, and the microcontroller accesses the corresponding region to obtain the temperature data. The temperature data is not in base 10, so it cannot be used directly for liquid crystal display, but also to be converted.

\section{SYSTEM PERFORMANCE TEST}

When the environment temperature of $15{ }^{\circ} \mathrm{C}$ test, each test time is 90 minutes, test a total of three times, take the average temperature as the chart data. First set the temperature upper and lower limit and the running time. Due to the low initial temperature, the fan is running at full speed, and the 1602 display shows the current temperature value and fixed time value. As the temperature rises, one of the heating fans closes. The room temperature rises slowly. When reach the set value $25{ }^{\circ} \mathrm{C}$, stop heating. The following figure 7 shows the indoor temperature when the heater is running, and the temperature data is taken from the DS18B20 temperature sensor.

In began to run after a period of time, temperature, growth rate of around $20{ }^{\circ} \mathrm{C}$ is reduced, when arrived at $25{ }^{\circ} \mathrm{C}$ near stop growing and gradually decreases, and then swings around $25{ }^{\circ} \mathrm{C}$. The change in the rate of temperature rise comes from the automatic switch of the output gear. The basic function test passed, and the physical model is shown in figure 8 below.

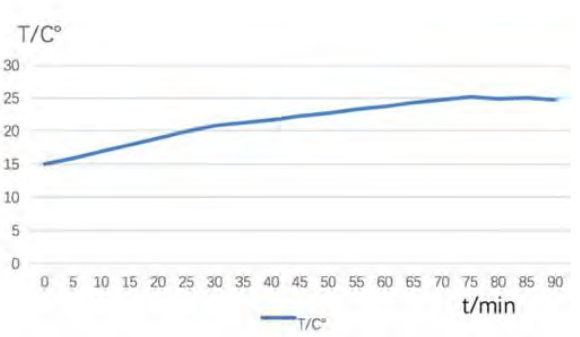

Fig. 7. Indoor real-time temperature

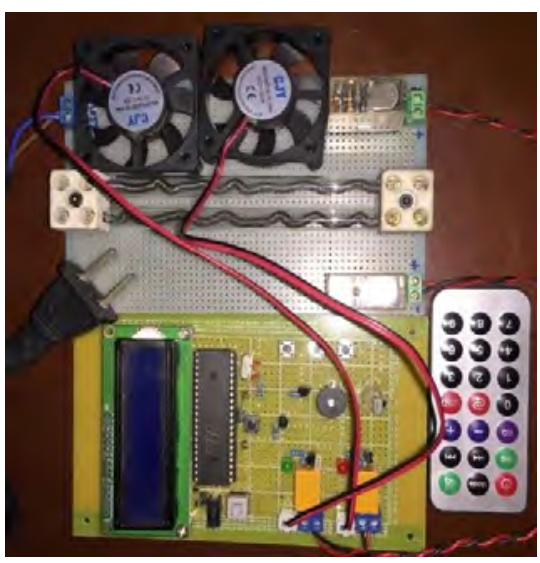

Fig. 8. A mock-up of the heater

\section{CONCLUSION}

This system has certain practical application value. The temperature range control system also needs to be improved, for example, the fan stepless speed control function can be added to make the 
heater work better. In addition, this heater is mainly for the household sector. If you want to use it in industry, you need to design it in combination with relevant standards.

\section{REFERENCE}

[1] Li Yangdong. Fuel and combustion analysis of thermal power boiler in power plant [J]. Jiangxi building materials, 2014, (20): 206 207.

[2] Zhang Junmo. Advanced tutorial on SCM - principles and applications (2nd edition) [M]. Beijing: Beijing University of Aeronautics and Astronautics Press, 2006.

[3] Wang Lijuan, Wang Yan. Multichannel temperature tester based on DS18B20 [J]. Manufacturing automation, 2013, (02): $129 \sim 133$.

[4] Gao Feng. Principle and interface technology of single-chip microcomputer [M]. Science Press 2007, 7782.

[5] Liu Aihua, Man Baoyuan. Sensor principle and application technology $[\mathrm{M}]$. Civilian posts and telecommunications press2010, $103 \sim 115$.
[6] Shen Hongwei. Design example and analysis of application system of single chip microcomputer [M]. Beijing university of aeronautics and astronautics press, 2001(7): $10 \sim 12$.

[7] Zhang Yigang. Principle and application of single chip microcomputer $[\mathrm{M}]$. Beijing: advanced education press, 2001, 397 407.

[8] Liang Yulin. Analytical relay.[J]. Business manager, 2013, (22): $352 \sim 372$.

[9] Shen Renyuan, Wu Yong. Simple manual for common electronic components [J]. Machine press 2006, 23 34.

[10] Xu Shuhua, Cheng Tuian, Yao Wansheng. Principle and application of microcomputer [M]. Harbin: Harbin Institute of Technology press, 1994, 85.

[11] Zhou Liangquan, Fu Enxi, Qi Shixin. Fundamentals of analog electronics technology (2nd edition) [M]. Higher Education Press,2004, 79 92.

[12] Chen Yongfu. Infrared detection and control circuit [M]. People's posts and telecommunications press 2003, 110 117.

[13] Zhu Min. Simulation design of infrared communication system based on $51 \mathrm{MCU}$ [J]. Mobile information, 2015, (02): $10 \sim 11$. 